\title{
NEUE ALTFUNDE GEHENKELTER RÖMISCHER SOLIDI DES KAISERS VALENS AUS TRANSKARPATIEN (UKRAINE)
}

\author{
P ÉTER PROH ÁSZKA
}

\begin{abstract}
Old New Roman Solidi of Valens with Suspension Loop from Transcarpathia (Ukraine). The article deals with two Roman gold coins of Valens, which were found in Transcarpathia. Only reports are about them in the Archive of the Hungarian National Museum. They were found in Užhorod and Nagyszlatina. Both coins have a suspension loop. Thanks to the descriptions it was possible to evaluate the finds. They belong to the evidences of the Roman political relationship with the German elites.
\end{abstract}

Keywords: Ukraine, Transcarpathia, Roman Period, solidi of Valens, coins with suspension loop.

Die Freilegung und Bearbeitung des germanischen Fürstengrabes von Poprad-Matejovce ist eng mit Karol Pieta verbunden (Pieta 2009). Beim Grabinventar befand sich eine Beigabe, die auch für die Datierung eine wichtige Rolle spielt. Es handelt sich um einen Solidus (Abb. 1) von Kaiser Valens, Typ RIC IX 49a. Die Münze wurde zwischen 9. August 375 und 25. August 383 in Trier geprägt und gibt den terminus post quem der Bestattung an. Der Solidus wurde nicht als Grabobolus ins Grab gelegt, sondern mit der angelöteten Öse als Schmuck getragen. Die Goldmünzen erscheinen ab der ersten Hälfte des 3. Jh. wieder in größerer Zahl im Karpatenbecken (Prohászka 2004), wobei jedoch die Zahl der Prägungen der einzelnen Kaiser nach Zeit und Gebiet differiert (Prohászka 2009). Sie zeigen mit ihrer Verbreitung auch die Bedeutung der Gentes bzw. ihre Siedlungsgebiete in der römischen Außenpolitik an. In der zweiten Hälfte des 3. Jh. kommen die Goldmünzen in besonders hoher Zahl vor, aber

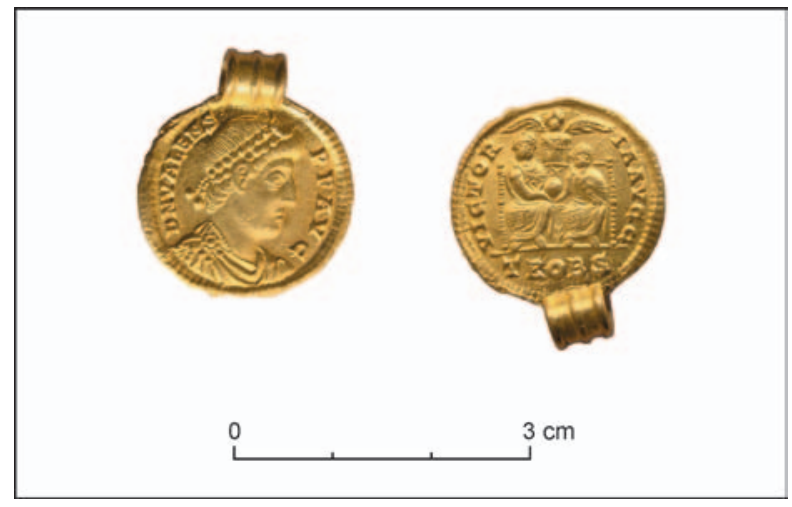

Abb. 1. Der Solidus aus dem Grab von Poprad-Matejovce. sie sind auch noch im 4 . Jh. charakteristisch. Die Münzen kamen jedoch überwiegend als Streufunde zum Vorschein, und nur wenige stammen aus Gräbern (Osztrópataka/Ostrovany, MadarasHalmok, Poprad-Matejovce). Von den Solidi des Kaisers Valens sind nur wenige Exemplare aus dem Karpatenbecken bekannt (Gabler 1975, 66, 67; Prohászka 2011a; 2011b). Wir möchten hier ihre Zahl um zwei Altfunde vermehren, über die jedoch nur archivalischen Angaben zur Verfügung stehen. Die Archivalien stellen eine wichtige und kaum genutzte Quelle dar, obwohl sich in Akten, Briefen und Notizen immer wieder Beschreibungen, Zeichnungen oder Abgüsse zahlreicher römischer Goldmünzen finden. Die Angaben zu den hier dargelegten Solidi befinden sich im Archiv des Ungarischen Nationalmuseums.

\section{Nagyszlatina (1900), heute Nižne Šolotvino, Ukraine}

Fundumstände: Streufund. Der Apotheker des Dorfes Szerednye János Speck sandte eine römische Goldmünze an das Nationalmuseum, die „Ung megye, Nagy Szlatina község határában, a föld müvelése alkalmával találtatott.“ - „An der Grenze des Dorfes Nagy Szlatina, Komitat Ung, beim Ackerbau gefunden wurde." Speck erkundigte sich nach der Bestimmung bzw. dem Wert der Münze. Nach der Abschrift des Antwortbriefes von József Hampel gehörte die eingesandte Goldmünze zu Kaiser Valens und wurde im 4. Jh. n. Chr. geprägt. Sie gehörte zu den gewöhnlichen Typen. Eine Skizze Hampels von der Münze findet sich auf dem Blatt, die auch eine Öse über der Kaiserbüste zeigt (Abb. 2).

Die Münze: Solidus des Valens, mit einer Öse versehen.

Emission: Konnte nicht bestimmt werden.

Verbleib/Literatur: Unbekannt - Ungarisches Nationalmuseum, Archiv des Münz- und Antikenkabinettes $660 / 1900$. 


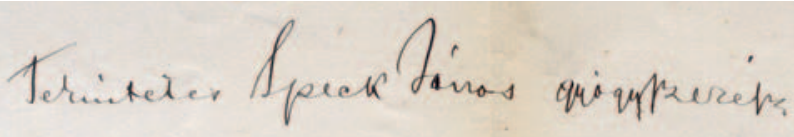

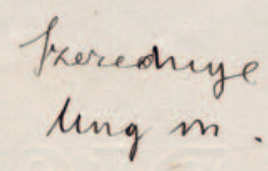

A bekildöx arangerem Valem

cafraitioil Malo' is Kefrieis

a Thi.... IV. Frizadban]

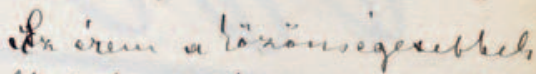

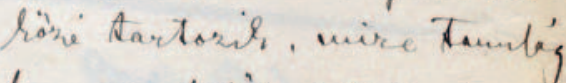

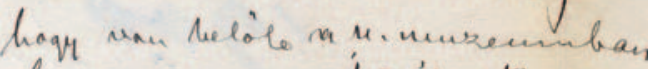

haviam darab. Fride towis

pelie ho Thoronaire tehetos

Telges dimteletrel

Pontanestiquo. Fetiober in

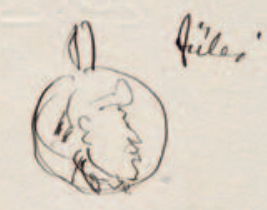

Abb. 2. József Hampels Zeichnung des gehenkelten Solidus von Nagyszlatina/Nižne Šolotvino (UNM Archiv).

Tab. I. Katalog der Solidi des Kaisers Valens (364-378) im Karpatenbecken.

\begin{tabular}{|c|c|c|c|}
\hline 1. & Iža (Slowakei) & Antiochia, RIC 2d (364-367) & Ondrouch 1964, 147 \\
\hline 2. & Jászberény (Ungarn) & Sirmium, RIC 1b (364) & $\begin{array}{l}\text { UNM, Archiv des Münz- und } \\
\text { Antikenkabinettes. 230/1887 }\end{array}$ \\
\hline 3. & Poprad-Matejovce (Slowakei) & Trier, RIC 49a (375-383) $\boldsymbol{\Delta}$ & Pieta 2009 \\
\hline 4. & Nagyszlatina (Nižne Šolotvino, Ukraine) & Valens $\boldsymbol{\Delta}$ & S. im Beitrag \\
\hline 5. & Ormód (Brestov, Ukraine) & $\begin{array}{l}\text { Im Hort befanden sich zwei Solidi des Valens: } \\
\text { a) Sirmium, RIC 1b (364) • } \\
\text { b) Constantinopolis, RIC 3c (364-367) }\end{array}$ & $\begin{array}{l}\text { Kropotkin } 2005,123 ; \\
\text { Kuzsinszky } 1892\end{array}$ \\
\hline 6. & $\begin{array}{l}\text { Szilágysomlyó } \\
\text { (Şimleu Silvaniei) Schatz I. }\end{array}$ & $\begin{array}{l}\text { Im Hort befanden sich neben anderen Multipla und } \\
\text { Goldschmuck sieben Multipla des Valens: } \\
\text { a) Rom, RIC } 25 \text { (375-378) } \\
\text { b) Thessalonica, RIC 28 (375-378) } \\
\text { c) Thessalonica, RIC 28 (375-378) } \\
\text { d) Rom, RIC } 26 \text { (375-378) } \\
\text { e) Rom, RIC } 26 \text { (375-378) } \\
\text { f) Antiochia, RIC } 38 \text { (375-378) } \\
\text { g) Antiochia, RIC } 37 \text { (375-378) }\end{array}$ & Bursche 1998, 241-246 \\
\hline 7. & Szügy (Ungarn) & $\begin{array}{l}1837 \text { wurde ein Schatz, bestehend aus Goldmünzen } \\
\text { des Valentinianus I. und Valens sowie Goldstücken, } \\
\text { gefunden. Im Fund befanden sich mindestens } 15 \text { Solidi, } \\
\text { von denen manche gehenkelt oder gelocht waren. }\end{array}$ & Prohászka 2007 \\
\hline 8. & Titel (Serbien) & $\begin{array}{l}\text { Im zweiten Schatz (1879) befanden sich unter den } \\
\text { Goldmünzen auch Prägungen des Valens. Nur ein } \\
\text { Solidus wurde beschrieben: Trier, RIC 1c/4 (364-367). }\end{array}$ & Prohászka 2011a, 155 \\
\hline 9. & Ungvár (Užhorod, Ukraine) & Valens, Typ RIC 2 ム & S. im Beitrag \\
\hline
\end{tabular}

$\Delta$ - mit Öse; $\bullet$-gelocht 


\section{Ungvár (1930), heute Užhorod, Ukraine}

Fundumstände: Streufund. Béláné Hegedűs erkundigte sich in einem Brief nach dem Wert eines Solidus von Valens und einer Napoleon-Goldmünze aus dem Jahr 1859. In dem Brief beschrieb sie die Münze so: „az érem egyik oldala egy nemes, római arcélü fejet ábrázol egyik oldalán DN Valens, másik oldalán Pfagg betükkel, másik oldala egy férfialakot ábrázol, ki jobbjában valami hosszú lándzsafélét tart, baljában pedig egy gömbön egy lábon álló nöalakot, ki kezében valami szívalakú karikát emel magasra, egyik oldalán ,Restitutor', a másikon, Reipublicae' felírással. Az éremnek a római fej fölött füle van..." - „eine Seite der Münze zeigt einen adeligen, römischen Kopf, auf einer Seite steht DN Valens, auf der anderen Buchstaben mit Pfagg. Die andere Seite zeigt eine Männergestalt, die in ihrer Rechten irgendeine lange Lanze und in ihrer Linken eine Kugel hält, auf der eine auf einem Fuß stehende Frauengestalt steht, die in ihrer Hand einen herzförmigen Ring hält. Auf dieser Seite steht eine Inschrift mit, Restitutor' auf der einen und ,Reipublicae' auf der anderen Seite. Die Münze hat über dem römischen Kopf eine Öse..."

Die Münze: Solidus des Valens, mit Öse. Av: DN VALENS PF AVG, Büste mit Diadem nach rechts. Rv: RESTITVTOR REIPUBLICAE, der stehende Kaiser, in einer Hand labarum, in der anderen eine auf einem Globus stehende Victoria haltend.

Emission: Eine genauere Bestimmung ist nicht möglich, weil die Buchstaben der Prägestätte nicht beschrieben wurden. Er gehört in die Serie Typ RIC 2 (364-378).

Verbleib/Literatur: Unbekannt - Ungarisches Nationalmuseum, Archiv des Münzkabinettes 177/1930.

Aus dem Barbaricum des Karpatenbeckens kennen wir Goldmünzen des Valens (mit diesen neuen Stücken) von insgesamt neun Fundorten (Tab. 1). Manche befanden sich in den Hortfunden von Szügy, Titel (1879), Ormód/Brestov bzw. im Fund I von Szilágysomlyó. Die Münzen des Hortes von Szilágysomlyó waren jedoch Multipla bzw. Medaillons (Bursche 1998, 241-246). Die anderen drei Horte bestanden ausschließlich aus Solidi. Eine Münze befand sich im Grab von Poprad-Matejovce und vier Solidi des Valens wurden als Streufunde entdeckt.

Aus dem Teil des Barbaricums westlich vom Karpatenbecken ist nur ein Stück bekannt. Dieser Solidus [Coh. 31 = Ant RIC 2d (345-367)] wurde in Iža gefunden (Ondrouch 1964, 147), wo die römische Gegenfestung von Brigetio noch im 4. Jh. stand. Aus Brigetio kennen wir ebenfalls nur einen Solidus des Valens, der im südöstlichen Bereich des Legionslagers ans Tageslicht kam und zur Serie RIC 16 oder 22 (367-375) gehört. Er wurde ebenfalls in Antiochia geprägt (Berković-Borota 1885, 204). In Pannonien sind noch manche Antiochener Prägungen aus Carnuntum bekannt (Prohászka 2011b, 79, 80), die darauf deuten können, daß diese Münzen durch das Militär hierher gelangten. Höchstwahrscheinlich überschritt der Solidus aus Iža in ähnlicher Weise die Donau. Die meisten Solidi des Valens zeigen einen Schwerpunkt im nordöstlichen Bereich des Karpatenbeckens. Nördlich der Karpaten, in Südpolen, sind nur zwei Goldmünzen des Valens bekannt (Bodzek 2009, 162-167; Bursche 1996). Ein Medaillon kam in Przemyśl zum Vorschein, ein Solidus befand sich im Hort von Krasnystaw I. Über diese Münze stehen uns keine genaueren Angaben zur Verfügung (Bodzek 2009, 182, 183). Das 29,5 g schwere Medaillon, Typ RIC 37-38a-b (376), kam 1934 beim Pflügen in der Nähe des Flusses San ans Tageslicht (Bodzek 2009, 185).

Von den etwas mehr als ein Dutzend Solidi kann man nur die Hälfte bestimmen. Ein Solidus aus Jászberény gehört nach seiner Beschreibung in einem an das Nationalmuseum geschickten Brief zum Typ RIC $1 b$ und wurde in Sirmium (364) geprägt (UNM, Archiv des Münz- und Antikenkabinettes 230/1887). Ebenfalls zu diesem Typ gehört ein Solidus im Hort von Ormód/Brestov (Abb. 3), der gelocht war (Kuzsinszky 1892). Der andere Solidus von Valens in diesem Hort ist eine Prägung aus Konstantinopel, Typ RIC 3c (364-367). Die einzige bestimmbare Valens-Münze aus dem 1879 gefundenen Hort von Titel ist eine Trierer Prägung vom Typ RIC 1c/4 (364-367; Prohászka 2011a, 155). Der Solidus aus Ungvár/Užhorod gehörte zur Serie Typ RIC 2, allerdings wurde die Prägestätte nicht festgehalten. Die jüngste Münze, auch eine Trierer Prägung vom Typ RIC 49a, befand sich im Grab von Poprad-Matejovce (Pieta 2009). Sie wurde zwischen 375 und 378 geprägt. Ebenfalls aus diesen Jahren stammen die Multipla im Fund I von Szilágysomlyó. Drei wurden in Rom, zwei in Thessaloniki und zwei in Antochia geprägt (Bursche 1998, 241-246). Nach diesen Angaben gelangten in den 360er Jahren erstmals Solidi zu den Gentes jenseits der Donau, etwas später, um 375, erscheinen dann wieder die späteren Typen des Valens im Fundmaterial.

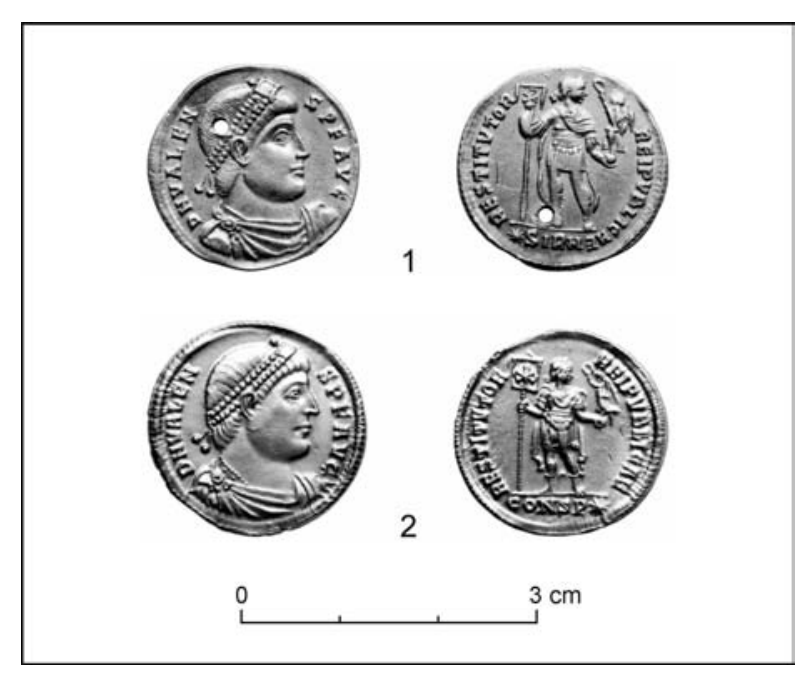

Abb. 3. Die beiden Solidi aus dem Hort von Ormód/Brestov. 


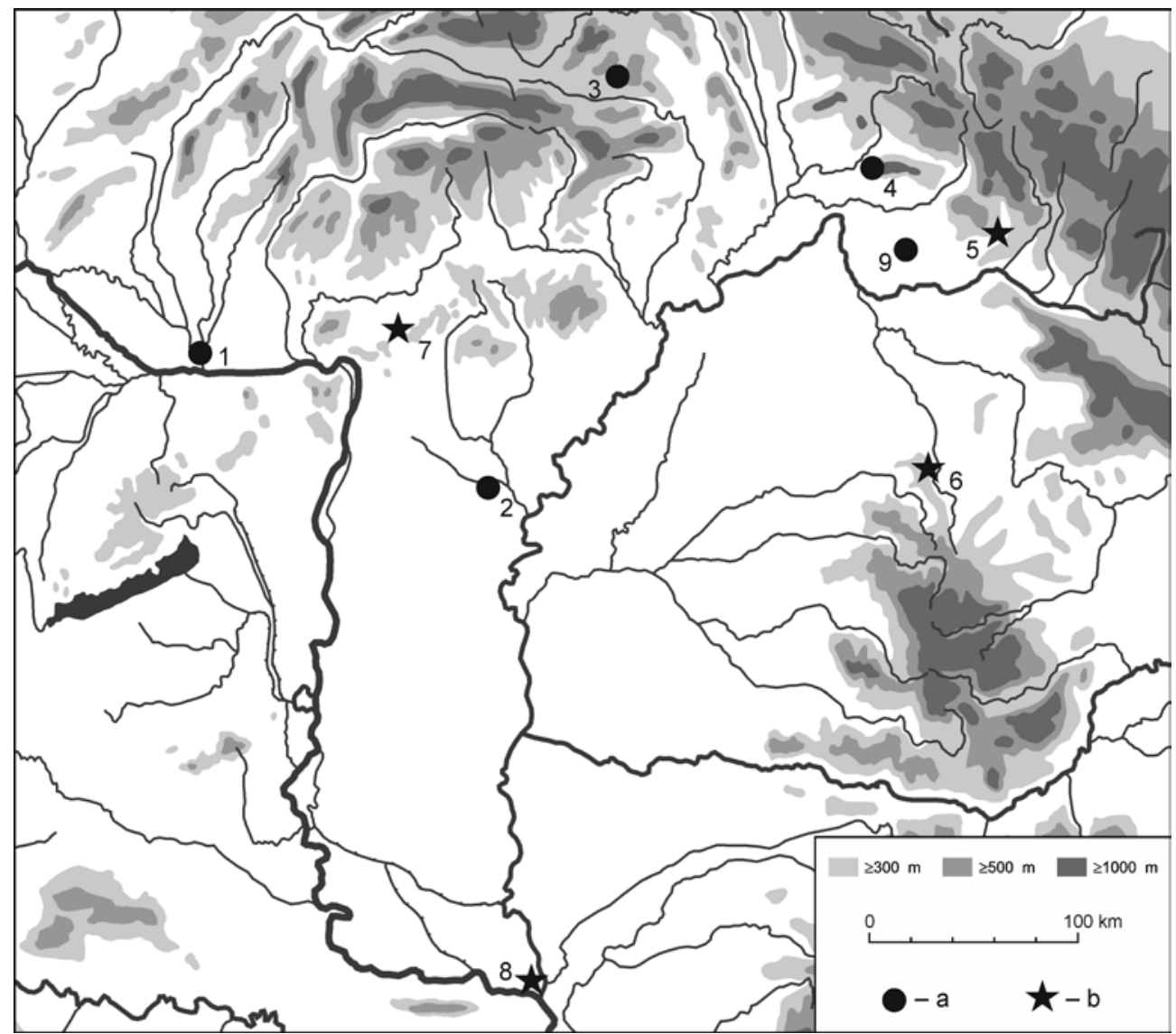

Abb. 4. Die Verbreitung der Goldmünzen des Valens im Karpatenbecken. 1 - Iža; 2 - Jászberény; 3 - Poprad-Matejovce; 4-Nagyszlatina/Nižne Šolotvino; 5 - Ormód/Brestov; 6-Szilágysomlyó/Şimleu Silvaniei; 7 - Szügy; 8 - Titel; 9 - Ungvár/ Užhorod. Legende: a - Einzelfund; b - Hortfund.

Bis auf die Münze im Hort von Titel (1879) erscheinen die Goldmünzen nördlich der Linie Szolnok - Nagyvárad/Orodea, hauptsächlich im nordöstlichen Teil des Karpatenbeckens (Abb. 4). In diesem Gebiet kommen auch die Solidi der Nebenkaiser von Valens vor (Prohászka 2011a, 162, 163). Seit der zweiten Hälfte des 3. Jh. ist eine $\mathrm{Zu}$ nahme der Goldmünzen im Fundmaterial dieses Gebietes beobachtbar, obwohl der Goldmünzverkehr unterschiedliche Intensivität zeigt. Trotzdem kann man von einem fast kontinuierlichen „Geldverkehr" sprechen, was man mit der spezifischen politischen Rolle der hier angesiedelten Völker zu erklären versuchte (Bóna 1986, 60). Welche Kontakte bzw. Ereignisse aber dahinterstanden, darüber stehen uns keine Angaben zur Verfügung (Stallknecht 1969). Diese Völker können die Goldmünzen nur von der kaiserlichen Regierung erhalten haben, welche die Münzen als Instrumente der römischen Sicherheits- bzw. Außenpolitik nutzte (Bemmann 2003, 58-62).

Die Solidi bzw. Multipla hatten bei den germanischen Völkern in der jüngeren römischen
Kaiserzeit eine sekundäre Verwendung gefunden, weil sie gehenkelt oder gelocht als Schmuck getragen wurden. Die größeren und schwereren Medaillons, wie zum Beispiel im Fund I von Szilágysomlyó, spielten - worauf Aleksander Bursche hinwies - bei der Legitimierung der germanischen Könige/Fürsten gewiß eine Rolle (Bemmann 2005, 31; Bursche 1998, 210-212; 1999, 221-228). Höchstwahrscheinlich trugen die Angehörigen der germanischen Oberschicht die Aurei/Solidi ebenfalls als Statussymbole (Bemmann 2005, 31). Ob dieser Brauch römische Vorbilder hatte oder sich bei den Germanen herausgebildet hat, kann man nicht sicher entscheiden. Gewiß wurden ab dem 3. Jh. im Römischen Reich gefaßte Münzen als Schmuck getragen (Prohászka 2004, 47). Dieser Brauch erscheint im Barbaricum ab der zweiten Hälfte des 3. Jh. und ist nicht nur im Karpatenbecken, sondern auch im europäischen Barbaricum bis zum 5. Jh. zu beobachten. Mehr als die Hälfte der Goldmünzen von Valens aus dem Karpatenbecken haben eine Öse oder ein Loch. In den Hortfunden von Ormód/Brestov und Szügy 
befanden sich gehenkelte sowie gelochte Solidi (Kuzsinszky 1892; Prohászka 2007). Neben den zwei alten Funden war auch der Solidus im Grab von Poprad-Matejovce gehenkelt.

Das 5. Jh. brachte nicht nur zahlreiche Veränderungen in der politischen, ethnischen und wirtschaftlichen Lage, sondern auch bei der Verwendung der Goldmünzen im Karpatenbecken. Ihre Rolle als Statussymbol hörte auf, denn im Fundmaterial beläuft sich die Zahl der gelochten und gehenkelten Solidi kaum auf ein halbes Dutzend (Prohászka 2009).

\section{LITERATUR}

Bemmann 2003 - J. Bemmann: Romanisierte Barbaren oder erfolgreiche Plünderer? Anmerkungen zur Intensität, Form und Dauer des provinzialrömischen Einflusses auf Mitteldeutschland während der jüngeren Römischen Kaiserzeit und der Völkerwanderungszeit. In: A. Bursche/R. Ciolek (red.): Antyk i Barbarzyny. Księga dedykowana Profesorowi Jerzemu Kolendo. Warszawa 2003, 55-108.

Bemmann 2005 - J. Bemmann: Zur Münz- und Münzersatzbeigabe in Gräbern der Römischen Kaiserzeit und Völkerwanderungszeit des mittel- und nordeuropäischen Barbaricums. In: H.-J. Häßler (Hrsg.): Neue Forschungsergebnisse zur nordwesteuropäischen Frühgeschichte unter besonderer Berücksichtigung der altsächsischen Kultur im heutigen Niedersachsen. Studien zur Sachsenforschung 15. Oldenburg 2005, 1-62.

Berković-Borota 1885 - M. Berković-Borota: Das Standlager Bregätium der Legio I Adjutrix, pia fidelis. Römerfunde und Ausgrabungen beim Baue der Positions-Batterie nächts Ó-Szőny bei Komorn. Österreichische Militärische Zeitschrift 4, 1885, 177-204.

Bodzek 2009 - J. Bodzek: Remarks in the Inflow of Roman Coins into Southern Poland in the Second Half of the $4^{\text {th }}$ and in the $5^{\text {th }}$ Centuries A.D. In: M. Woloszyn (ed.): Byzantine Coins in Central Europe between the $5^{\text {th }}$ and $10^{\text {th }}$ Century. Moravia Magna. Seria Polona. Vol. III. Kraków 2009, 155-204.

Bóna 1986 - I. Bóna: Szabolcs-Szatmár megye régészeti emlékei. In: G. Entz (szerk.): Szabolcs-Szatmár megye müemlékei I. Budapest 1986, 15-91.

Bursche 1996 - A. Bursche: Later Roman-Barbarian Contacts in Central Europe Numismatic Evidence. Studien zur Fundmünzen der Antike 11. Berlin 1996.

Bursche 1998 - A. Bursche: Ztote medaliony rzymskie w Barbaricum. Symbolika prestiżu i władzy społeczeństw barbarzyńskich u schytku starożytności. Warszawa 1998.

Bursche 1999 - A. Bursche: Die Rolle römischer Goldmedaillone in der Spätantike. In: W. Seipel (Hrsg.): Barbarenschmuck und Römergold. Der Schatz von Szilágysomlyó. Wien 1999, 39-54.
Gabler 1975 - D. Gabler: Zur Fragen der Handelsbeziehungen zwischen den Römern und den „Barbaren” im Gebiet östlich von Pannonien. In: H. Grünert/H.-J. Dölle (Hrsg.): Römer und Germanen in Mitteleuropa. Berlin 1975, 87-121.

Kropotkin 2005 - V. V. Kropotkin: Les trouvailles de monnaies romaines en U.R.S.S. Moneta 49. Wetteren 2005.

Kuzsinszky 1892 - B. Kuzsinszky: Az ormódi aranykincs érmei. Archaeologiai Értesítő 12, 1892, 225-338.

Ondrouch 1964 - V. Ondrouch: Nálezy keltských, antických a byzantských mincí na Slovensku. Bratislava 1964.

Pieta 2009 - K. Pieta: Das germanische Fürstengrab aus Poprad-Matejovce. In: U. von Freeden/H. Friesinger/E. Wamers (Hrsg.): Glaube, Kult und Herrschaft. Phänomene des Religiösen im 1. Jahrtausend n. Chr. Akten des 59. Internationalen Sachsensymposions und der Grundprobleme der frühgeschichtlichen Entwicklung im Mitteldonauraum. Kolloquien zur Vor- und Frühgeschichte 12. Bonn 2009, 107-122.

Prohászka 2004 - P. Prohászka: Az osztrópatakai vandál királysír. Esztergom 2004.

Prohászka 2007-P. Prohászka: Der spätrömische Schatzfund von Szügy (1837). Numismatický sborník 22, 2007, 31-38.

Prohászka 2009-P. Prohászka: Ost- und Weströmische Goldmünzen aus dem 5. Jahrhundert im Karpatenbecken. In: M. Woloszyn (ed.): Byzantine Coins in Central Europe between the $5^{\text {th }}$ and $10^{\text {th }}$ Century. Moravia Magna. Seria Polona. Vol. III. Kraków 2009, 83-115.

Prohászka 2011a - P. Prohászka: Die spätrömischen Solidusfunde aus Titel (Serbien). Acta Archaeologica Carpathica 46, 2011, 145-170.

Prohászka 2011b - P. Prohászka: Bemerkungen zum spätrömischen und frühvölkerwanderungszeitlichen Goldmünzverkehr in Pannonia I und Valeria. In: O. Heinrich-Tamáska (Hrsg.): Keszthely-Fenékpuszta im Kontext spätantiker Kontinuitätsforschung zwischen Noricum und Moesia. Castellum Pannonicum Pelsonense 2. BudapestLeipzig 2011, 61-86.

Stallknecht 1969 - B. Stallknecht: Untersuchungen zur römischen Außenpolitik in der Spätantike (306-395 n. Chr.). Habelts Dissertationsdrucke. Alte Geschichte 7. Bonn 1969.

Mgr. Dr. Péter Prohászka, PhD.

Archeologický ústav SAV

Akademická 2

SK - 94921 Nitra

prohaszkapeter1975@gmail.com 
